\title{
Are Maxwell knots integrable?
}

\author{
A. Morozov ${ }^{1,2,3, a}$, N. Tselousov ${ }^{1,3, b}$ (i) \\ ${ }^{1}$ Institute for Theoretical and Experimental Physics, Moscow 117218, Russia \\ 2 Institute for Information Transmission Problems, Moscow 127994, Russia \\ ${ }^{3}$ Moscow Institute of Physics and Technology, Dolgoprudny 141701, Russia
}

Received: 4 December 2020 / Accepted: 9 December 2020 / Published online: 22 December 2020

(C) The Author(s) 2020

\begin{abstract}
We review properties of the null-field solutions of source-free Maxwell equations. We focus on the electric and magnetic field lines, especially on limit cycles, which actually can be knotted and/or linked at every given moment. We analyse the fact that the Poynting vector induces selfconsistent time evolution of these lines and demonstrate that the Abelian link invariant is integral of motion. We also consider particular examples of the field lines for the particular family of finite energy source-free "knot" solutions, attempting to understand when the field lines are closed - and can be discussed in terms of knots and links. Based on computer simulations we conjecture that Ranada's solution, where every pair of lines forms a Hopf link, is rather exceptional. In general, only particular lines (a set of measure zero) are limit cycles and represent closed lines forming knots/links, while all the rest are twisting around them and remain unclosed. Still, conservation laws of Poynting evolution and associated integrable structure should persist.
\end{abstract}

\section{Introduction}

Knots are playing an increasingly important role in mathematical physics, but in phenomenological physics they remain rather exotic. This is despite knots served as an inspiration to many great physicists, starting at least from Lord Kelvin [1]. In fact the reason can be simple: we are not looking around with enough attention to notice unconventional things. From this perspective, it was a great breakthrough when in 1989 Ranada demonstrated [2,3] that Hopf link appears naturally in a solution to the ordinary Maxwell equations, i.e. is no less natural than the conventional plane wave solution. In this paper we are going to discuss some surprising properties of such knot-revealing solutions and mention some open problems - without going into important

\footnotetext{
a e-mail: morozov.itep@mail.ru (corresponding author)

be-mail: tselousov.ns@phystech.edu
}

but relatively sophisticated mathematics, like in [4] or [5,6]. We begin in Sect. 2 from consideration of the field lines of electric and magnetic fields. When they are closed, they can be knotted or linked. A given field line evolves in time this evolution appears to proceed along the Poynting vector field, what, however imposes a non-trivial and non-expected condition on the parametrization - we discuss these issues in Sects. 3 and 3.3. Moreover, closed and linked field lines remain linked, and this is guaranteed by the conservation laws provided by knot invariants - which in this context serve as equations of motion and can even make the evolution of field lines integrable - see Sects. 4 and 5. We illustrate this general consideration by analysis of the Ranada's Hopf solution in Sect. 6.1 and by a brief discussion of more general solutions in Sect. 6.2. In Hopf solution all field lines are closed but unknotted, and every pair of field lines forms a Hopf link with no non-trivial links of higher order. However, in other solutions generic field lines are not closed, only some are but they can form quite non-trivial knots and links. There is still a question, which knots and links can appear and how abundant they are - see [5] for a possible approach to this problem. We end with a brief summary in Sect. 7.

\section{Field lines at a given time moment}

In this paper we review properties of solutions of Maxwell equations:

$$
\begin{array}{ll}
\frac{\partial \mathbf{B}}{\partial t}+\nabla \times \mathbf{E}=0, & \nabla \cdot \mathbf{B}=0 ; \\
\frac{\partial \mathbf{E}}{\partial t}-\nabla \times \mathbf{B}=0, & \nabla \cdot \mathbf{E}=0 .
\end{array}
$$

In particular, we are interested in the topological structure of the field lines so let us define the field line itself. To be more specific, let us concentrate only on the electric field lines, however our analysis can be carried out for the magnetic field 
lines analogously. Let $\mathbf{E}$ be an electric field at a given moment of time and $\mathbf{x}(s)$ - a smooth curve in some parametrization $s$. Then $\mathbf{x}(s)$ is called an electric field line if the following condition holds for any point on the curve:

$$
\frac{d \mathbf{x}(s)}{d s} \uparrow \uparrow \mathbf{E}(\mathbf{x}(s))
$$

In other words, the tangent vector of the curve should be collinear to the electric field at each point on the curve. We note that the parametrization of the field line is ambiguously defined. To proceed with practical calculation, we will fix a parametrization that is suitable for our purposes. For example, one may consider a field line in different parametrizations:

$$
\frac{d \mathbf{x}\left(s_{1}\right)}{d s_{1}}=\mathbf{E}\left(\mathbf{x}\left(s_{1}\right)\right) \frac{d \mathbf{x}\left(s_{2}\right)}{d s_{2}} \frac{\mathbf{E}\left(\mathbf{x}\left(s_{2}\right)\right)}{\left|\mathbf{E}\left(\mathbf{x}\left(s_{2}\right)\right)\right|}=\frac{d \mathbf{x}\left(s_{3}\right)}{d s_{3}}=\frac{\mathbf{E}\left(\mathbf{x}\left(s_{3}\right)\right)}{\left|\mathbf{E}\left(\mathbf{x}\left(s_{3}\right)\right)\right|^{2}} \ldots
$$

All these equations define the same field line. The second parametrization $s_{2}$ corresponds to the length of the field line, but we mainly use the third one $s_{3}$ in our analysis of the knot invariants.

At a given moment of time there are different types of field lines. The force lines with endpoints are prohibited by the zero divergence condition. In other words, endpoints of the force lines are electric/magnetic charges, which are absent in our case. Generally, several types are available: closed loops of finite length and endless curves of infinite length. The curves with an infinite length may go to infinity or be located in the finite area.

Closed field lines are in fact limit cycles. It would be interesting to find sufficient conditions of closedness of the field lines. We discuss particular examples of field lines in Sects. 6.1 and 6.2.

\section{Time evolution of force lines}

In our paper we consider only null-field solutions of Maxwell equations. This means that at every moment of time and at every point in space the following conditions hold:

$$
\mathbf{E} \cdot \mathbf{B}=0, \quad \mathbf{E}^{2}=\mathbf{B}^{2} .
$$

Generally, Maxwell equations do not preserve these conditions, which makes them non-trivial. However, such solutions exist and a procedure of constructing them was described in detail in s.7 of [6]. The simplest solution of this kind is the Ranada's Hopf solution and we discuss it in Sect. 6.1.

We mainly focus on the following property of null-field solutions. It turns out that one can define self-consistent time evolution of the field lines [7,8]. Generally, at different moments of time the space is filled with different field lines, due to the electric field evolution according to Maxwell equations. To give a prescription of how a field line deforms and moves in space, one needs to define a velocity vector $\mathbf{v}$ for each point of a field line. This vector $\mathbf{v}$ is normal to the field line at each point. This structure will match the field lines at different moments of time.

However, to define the time evolution of the field lines, a velocity vector field should obey a very special, yet natural condition. The condition requires that any two points of a field line become points of another field line in the further moment of time when moved along the velocity vector field (see Fig. 1). In other words, points of a field lines can not become points of different field lines. Alternatively, one could require that the following curve

$\mathbf{x}^{\prime}(s)=\mathbf{x}(s)+\mathbf{v}(\mathbf{x}(s)) d t$

is a field line that is defined at the moment $t+d t$.

In case of null-field solutions, the velocity vector $\mathbf{v}$ can be chosen as the normalized Poynting vector:

$\mathbf{p}=\frac{\mathbf{E} \times \mathbf{B}}{|\mathbf{E}||\mathbf{B}|}$

\subsection{Poynting time evolution}

In this section we demonstrate that the Poyting vector in a null-field solution induces self-consistent time evolution of the force lines. To do this, we explicitly check the selfconsistency of the condition from the previous section.

We consider an electric field line $\mathbf{x}(s)$ of a null-field solution at the moment $t$. In this section we fix the parametrization $s$ by the following equation:

$$
\frac{d \mathbf{x}(s)}{d s}=\mathbf{E}(\mathbf{x}(s), t)
$$

Fig. 1 The picture demonstrates the condition that the vector field $\mathbf{v}$ should obey to define self-consistent time evolution of the field lines. For any two points on a field line at the moment $t$ the ends of the vectors $\mathbf{v} d t$ at the corresponding points lie on a field line that is defined at the moment $t+d t$

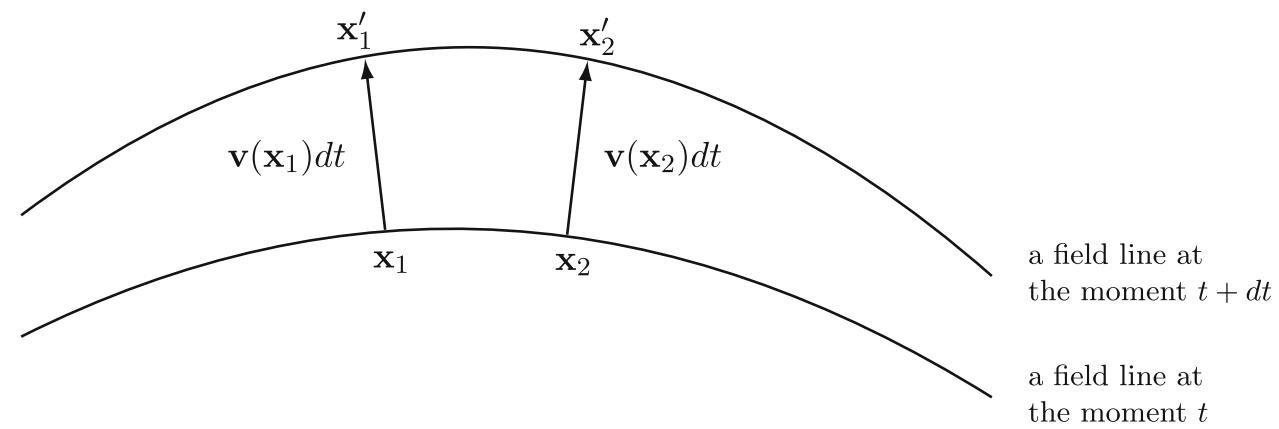


Then we consider an auxiliary line $\mathbf{x}^{\prime}(s)$ that is obtained from $\mathbf{x}(s)$ by a slight shift along the Poynting vector $\mathbf{p}$ :

$\mathbf{x}^{\prime}(s)=\mathbf{x}(s)+\mathbf{p}(\mathbf{x}(s), t) d t$

Note that we shift along the Poynting vector by $d t$. The main claim is that $\mathbf{x}^{\prime}(s)$ coincides as geometrical objects with some field line at the moment $t+d t$. Namely, a tangent vector of the line $\mathbf{x}^{\prime}(s)$ is collinear to the electric field at the moment $t+d t$, it means that they define the same field line:

$\frac{d \mathbf{x}^{\prime}(s)}{d s} \uparrow \uparrow \mathbf{E}\left(\mathbf{x}^{\prime}(s), t+d t\right)$

We should note that this collinearity holds only in the first order in $d t$. To explicitly see this, we compute the corresponding cross product:

$$
\begin{aligned}
& \frac{d \mathbf{x}^{\prime}(s)}{d s} \times \mathbf{E}\left(\mathbf{x}^{\prime}(s), t+d t\right)=(\mathbf{E}+(\mathbf{E} \cdot \nabla) \mathbf{p} d t) \\
& \quad \times\left(\mathbf{E}+(\mathbf{p} \cdot \nabla) \mathbf{E} d t+\frac{\partial \mathbf{E}}{\partial t} d t+o(d t)\right)= \\
& =d t \mathbf{E} \times\left((\mathbf{p} \cdot \nabla) \mathbf{E}-(\mathbf{E} \cdot \nabla) \mathbf{p}+\frac{\partial \mathbf{E}}{\partial t}\right)+o(d t)=o(d t)
\end{aligned}
$$

To simplify the formulas, we omit the explicit coordinate and time dependence of the fields meaning $\mathbf{E}=\mathbf{E}(\mathbf{x}(s), t)$ and $\mathbf{p}=\mathbf{p}(\mathbf{x}(s), t)$. We compute the expression in the brackets using the following identity for null-field solutions:

$\mathrm{E}_{\beta} \mathrm{p}_{\alpha}-\mathrm{E}_{\alpha} \mathrm{p}_{\beta}=\epsilon_{\alpha \beta \gamma} \mathrm{B}_{\gamma}$

Taking the derivative of (11) and using the Maxwell equations, we obtain the expression of the form:

$(\mathbf{p} \cdot \nabla) \mathbf{E}-(\mathbf{E} \cdot \nabla) \mathbf{p}+\frac{\partial \mathbf{E}}{\partial t}=-(\nabla \cdot \mathbf{p}) \mathbf{E}$

The r.h.s is collinear to the electric field $\mathbf{E}$ that ensures (10). Finally, we conclude that the Poynting vector defines the self-consistent time evolution of the field line. Therefore, we can think of a field line as a strand where each point moves with the velocity vector $\mathbf{p}$. From now we understand the time dependence of the field lines $\mathbf{x}(s, t)$ as it is induced by the Poynting vector:

$\frac{d \mathbf{x}(s, t)}{d t}=\mathbf{p}(\mathbf{x}(s, t), t)$

It is evident now why we consider the normalized Poyting vector. If one chooses another normalization for the Poynting vector it will spoil (12).

\subsection{Examples}

For demonstrative purposes let us discuss plane wave nullfield solutions of Maxwell equations to analyse the structure of the field lines and its time evolution induced by the Poynting vector.

\section{- Plane wave with linear polarization}

The plane wave with linear polarization has the form:

$$
\begin{aligned}
& \mathbf{E}=\left(\begin{array}{c}
E \cos (z-t) \\
0 \\
0
\end{array}\right) \quad \mathbf{B}=\left(\begin{array}{c}
0 \\
E \cos (z-t) \\
0
\end{array}\right) \\
& \mathbf{p}=\left(\begin{array}{l}
0 \\
0 \\
1
\end{array}\right)
\end{aligned}
$$

Note that at a given moment of time the the electric field changes along the $z$-axis. However, all the electric field lines are straight lines parallel to the $x$-axis. The field lines fill the whole space except "singular" $x y$-planes with coordinates $z=t+\pi / 2+\pi n, n \in \mathbb{Z}$. We can write an explicit formula for the electric field line that goes through the point $\left(y_{0}, z_{0}\right)$ in the $y z$-plane at the moment $t_{0}$ :

$$
\mathbf{x}(s, t)=\left(\begin{array}{c}
s E \cos \left(z_{0}-t_{0}\right) \\
y_{0} \\
z_{0}+t-t_{0}
\end{array}\right)
$$

At each moment of time the tangent vector of the field line is the electric field:

$$
\begin{aligned}
& \frac{d \mathbf{x}(s, t)}{d s}=\mathbf{E}(\mathbf{x}(s, t), t) \\
& \Leftarrow \quad\left(\begin{array}{c}
E \cos \left(z_{0}-t_{0}\right) \\
0 \\
0
\end{array}\right)=\left(\begin{array}{c}
E \cos \left(z_{0}+t-t_{0}-t\right) \\
0 \\
0
\end{array}\right)
\end{aligned}
$$

We note that the tangent vector of the particular field line does not change over time. This is due to the fact $\nabla \cdot \mathbf{p}=0$ and we discuss this in Sect. 3.3. The velocity of the points of the field line is the Poynting vector:

$$
\frac{d \mathbf{x}(s, t)}{d t}=\mathbf{p}(\mathbf{x}(s, t), t) \quad \Leftarrow \quad\left(\begin{array}{l}
0 \\
0 \\
1
\end{array}\right)=\left(\begin{array}{l}
0 \\
0 \\
1
\end{array}\right)
$$

The structure of the field lines flows along the $z$-axis over time as a whole and do not change. This is evident from the coordinate independence of the Poynting vector. 

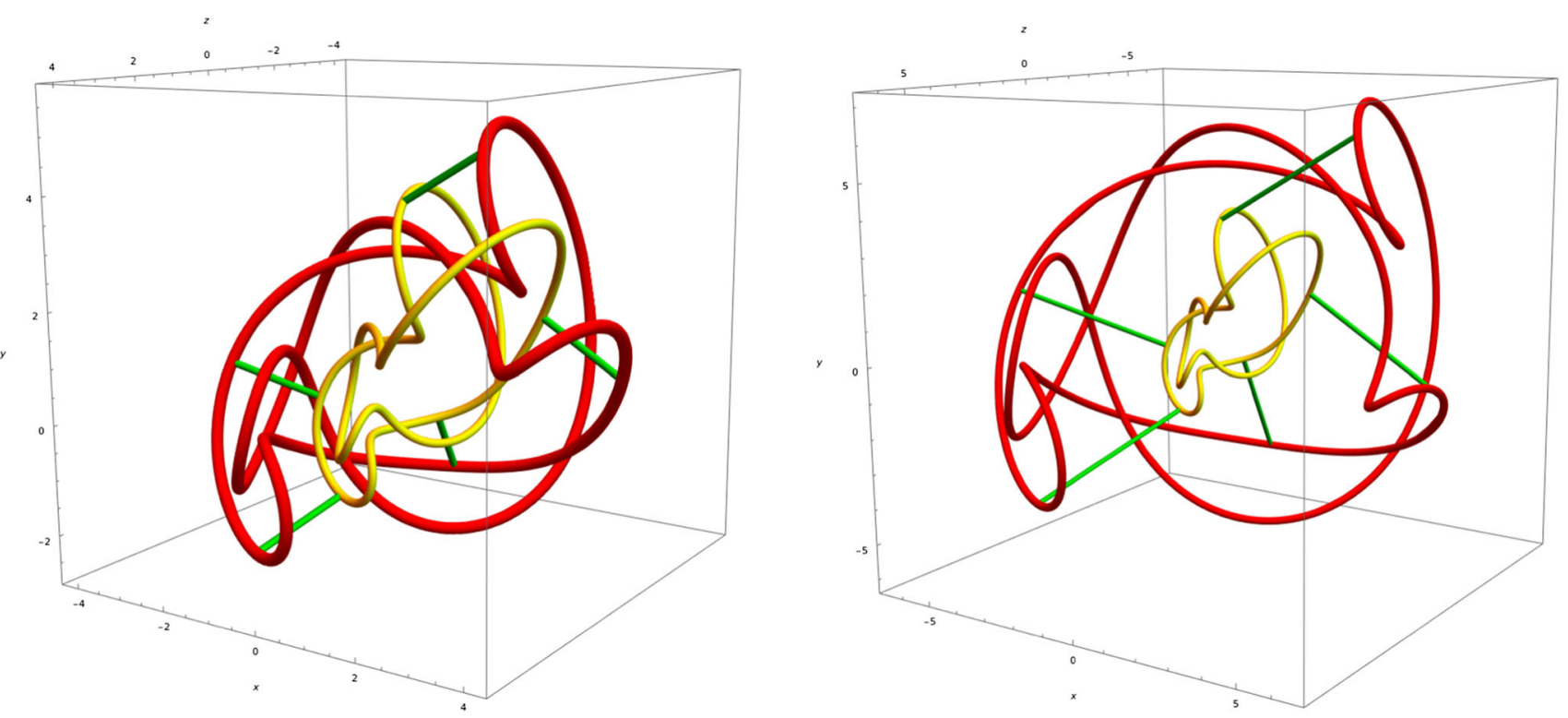

Fig. 2 The little yellow knot becomes the big red one under time evolution. There are pictures of two successive moments of time. The green curves are the trajectories. The topological structure of knot does not change under the time evolution

\section{- Plane wave with elliptic polarization}

This solution has the form:

$$
\begin{gathered}
\mathbf{E}=\left(\begin{array}{c}
E_{x} \cos (z-t) \\
E_{y} \sin (z-t) \\
0
\end{array}\right) \quad \mathbf{B}=\left(\begin{array}{c}
-E_{y} \sin (z-t) \\
E_{x} \cos (z-t) \\
0
\end{array}\right) \\
\mathbf{p}=\left(\begin{array}{l}
0 \\
0 \\
1
\end{array}\right)
\end{gathered}
$$

This solution of Maxwell equations has a slightly more complicated structure of the field lines, however the time evolution is the same as in the previous example - the field lines move along the $z$-axis, while the structure of the field lines remains the same. The explicit formula for the field line that goes through the point $\left(x_{0}, y_{0}, z_{0}\right)$ at the moment $t_{0}$ :

$\mathbf{x}(s, t)=\left(\begin{array}{c}x_{0}+s E_{x} \cos \left(z_{0}-t_{0}\right) \\ y_{0}+s E_{y} \sin \left(z_{0}-t_{0}\right) \\ z_{0}+t-t_{0}\end{array}\right)$

The field lines are normal to the $z$-axis and all field lines on a particular $x y$-plane are parallel. However, the direction of the field lines at a fixed moment of time is continuously changing along the $z$-axis. Namely, the direction rotates clockwise or anticlockwise along the $z$-axis at a fixed moment of time depending on the mutual sign of $E_{x}, E_{y}$.
At each moment of time the tangent vector is the electric field and the velocity is the Poynting vector:

$$
\begin{aligned}
\frac{d \mathbf{x}(s, t)}{d s}=\mathbf{E}(\mathbf{x}(s, t), t) & \\
\Leftarrow\left(\begin{array}{c}
E_{x} \cos \left(z_{0}-t_{0}\right) \\
E_{y} \sin \left(z_{0}-t_{0}\right) \\
0
\end{array}\right)= & \left(\begin{array}{c}
E_{x} \cos \left(z_{0}+t-t_{0}-t\right) \\
E_{y} \sin \left(z_{0}+t-t_{0}-t\right) \\
0
\end{array}\right) \\
\frac{d \mathbf{x}(s, t)}{d t}=\mathbf{p}(\mathbf{x}(s, t), t) & \Leftarrow\left(\begin{array}{l}
0 \\
0 \\
1
\end{array}\right)=\left(\begin{array}{l}
0 \\
0 \\
1
\end{array}\right)
\end{aligned}
$$

\section{- Electromagnetic knot}

We consider a member of the family of finite energy source-free knot solutions of Maxwell equations [5,6]. We do not provide the explicit form of the electric and magnetic fields, because its is too complicated. The crucial point is that we use the null-field solution. Instead of formulas we provide a computer simulation. We consider a closed field lines - limit cycles, that represent the trefoil knots. The little yellow knot becomes the big red one under the time evolution (see Fig. 2). The green curves are the trajectories of points and obey equation (13).

We argue that this picture represents general features of the Poynting evolution:

1. The Poynting evolution is well defined. The green trajectories ensure the self-consistency condition. 


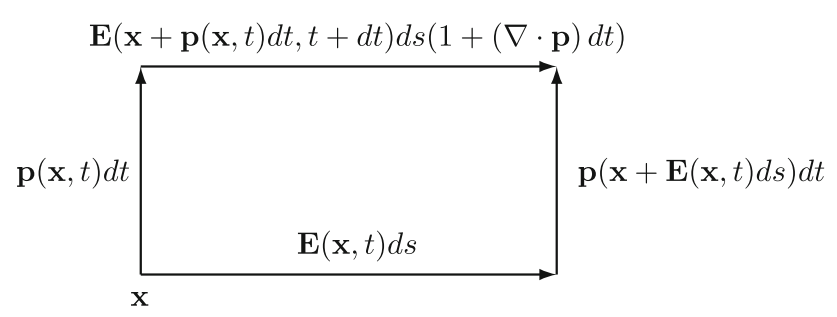

Fig. 3 Graphical interpretation of the self-consistency condition. The tangent vector of the auxiliary line is collinear to the electric field

2. Limit cycles remain limit cycles. It is a strong argument in support of the Poynting evolution, because the time evolution of the limit cycles is well defined without any additional structure. Indeed, generally limit cycles are distinguished field lines, because they are closed. The Poynting time evolution coincides with the evolution of the limit cycles.

3. The topological structure of the limit cycles remains the same. Indeed, in both cases (yellow and red) the knot is the trefoil knot $3_{1}$.

\subsection{Peculiarities of Poynting evolution}

In this section we derive the parametrization of the field lines that is suitable for our further analysis of the link invariants. Also we argue that in this parametrization the commutativity of flows along $s$ and $t$ is evident.

As has been shown in the Sect. 3.1, the tangent vector of the auxiliary line

$\mathbf{x}^{\prime}(s)=\mathbf{x}(s)+\mathbf{p}(\mathbf{x}(s), t) d t$

is not equal to the electric field at the moment $t+d t$ but only collinear. Graphically it can be shown in the following parallelogram of vectors 3 .

This parallelogram is the consequence of the formula (12). As can be seen, the upper vector is a slightly rescaled electric field. This fact can be thought of as the change in the parametrization:

$d s^{\prime}=d s(1+(\nabla \cdot \mathbf{p}) d t)$

We are free to multiply the electric field by a function $f\left(\mathbf{E}^{2}\right)$ and the corresponding field lines will remain the same. However, the function $f$ can be chosen in such a way that the parametrization does not change:

$d s^{\prime}=d s$

In other words, the tangent vector of the auxiliary line (the upper vector in the parallelogram) will be equal to the vector $\mathbf{E} f\left(\mathbf{E}^{2}\right)$ on the shifted line at the moment $t+d t$. Namely:

$$
\frac{d \mathbf{x}(s)}{d s}=\mathbf{E}(\mathbf{x}, t) f\left(\mathbf{E}^{2}(\mathbf{x}, t)\right)
$$

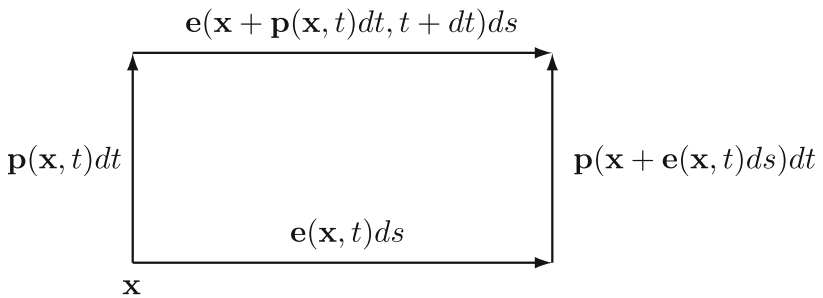

Fig. 4 Graphical interpretation of the self-consistency condition for rescaled fields. The tangent vector of the auxiliary line coincides with the electric field

$\frac{d \mathbf{x}^{\prime}(s)}{d s}=\mathbf{E}\left(\mathbf{x}^{\prime}, t+d t\right) f\left(\mathbf{E}^{2}\left(\mathbf{x}^{\prime}, t+d t\right)\right)$

From the last equation in the first order in $d t$ we obtain the following constraint on the function $f$ :

$(\nabla \cdot \mathbf{p})\left(f+\mathbf{E}^{2} \frac{\partial f}{\partial \mathbf{E}^{2}}\right)=0$

Note that if $(\nabla \cdot \mathbf{p})=0$, the equation (26) will be satisfied automatically with any function $f$. Otherwise, if $(\nabla \cdot \mathbf{p}) \neq 0$, the function is determined uniquely:

$f\left(\mathbf{E}^{2}\right)=\frac{1}{\mathbf{E}^{2}}$

To simplify the formulas, let us introduce the rescaled fields:

$\mathbf{e}:=\mathbf{E} / \mathbf{E}^{2}$

Note that the rescaling of the fields does not spoil the null field conditions (4). In these new notations both the analog of the formula (12) and the parallelogram 4 look simpler and do not contain the term with $(\nabla \cdot \mathbf{p})$ :

$(\mathbf{p} \cdot \nabla) \mathbf{e}+\frac{\partial \mathbf{e}}{\partial t}=(\mathbf{e} \cdot \nabla) \mathbf{p}$

This formula is crucial for our analysis of the topological structure of the field lines. The rescaling of the electric fields does not change the structure of the field lines, however the formulas and calculations in the new notations are much simpler.

As was discussed in the previous Sect. 3.1, each point of the field line moves over time with the velocity $\mathbf{p}$. Therefore, the system of the equations on the field lines takes the following form:

$\frac{d \mathbf{x}(s, t)}{d s}=\mathbf{e}(\mathbf{x}(s, t), t)$

$\frac{d \mathbf{x}(s, t)}{d t}=\mathbf{p}(\mathbf{x}(s, t), t)$

Using the equation (30), we obtain the following property:

$\frac{d \mathbf{e}(\mathbf{x}(s, t), t)}{d t}=\frac{d \mathbf{p}(\mathbf{x}(s, t), t)}{d s}$ 
This property ensures commutativity of flows along $s$ and $t$ in the chosen parametrization.

\section{Knot invariants as invariants of motion}

There are solutions of Maxwell equations that exhibit topologically nontrivial structure of the electric field lines that makes them worth studying. Firstly, some solutions have the property that all/some of the field lines are closed loops limit cycles. Secondly, a particular closed field line can be topologically nontrivial i.e. it can be a knot. Thirdly, a pair of closed field lines can be linked.

A closed field line could be identified with some string or strand. Each element of the string moves with velocity equal to the Poynting vector at the corresponding point of the space. As soon as one identifies the field lines with moving strands, natural questions appear. One can ask if the topological structure of the strands is preserved over time. Namely, if a strand was knotted, can it become a different knot? If a pair of strands was linked together, can they become unlinked at a further moment of time? These questions are quite similar and refer to the situation that involves crossings of strands.

There are tools to address this type of questions. They are called knot and link invariants. These invariants are preserved under smooth deformations of knots and links that do not admit crossings of lines. In our work we use the Gauss linking integral [9] which is an example of a link invariant:

$\operatorname{Link}_{\mathbf{x}, \mathbf{y}}=\oint d x_{\alpha} \oint d y_{\beta} \epsilon_{\alpha \beta \gamma} \frac{x_{\gamma}-y_{\gamma}}{|\mathbf{x}-\mathbf{y}|^{3}}$

We show that the linking integral computed for a pair of electric field lines does not change over time, i.e. it is an integral of the Poynting evolution. This fact gives the answer for the second question, while the answer for the first one involves non-Abelian generalization of the Gauss integral and we leave it for future studies.

In this section we consider an application of the Gauss linking integral to the study of the evolution of the field lines. We show that the linking integral applied for a pair of limit cycles is preserved under the time evolution of the field lines induces by the Poynting vector.

The linking integral is a topological invariant and it is defined for a pair of closed lines $\mathbf{x}\left(s_{x}\right), \mathbf{y}\left(s_{y}\right)$ :

$\operatorname{Link}_{\mathbf{x}, \mathbf{y}}=\int d s_{x} d s_{y} \epsilon_{\alpha \beta \gamma} \frac{d x_{\alpha}}{d s_{x}} \frac{d y_{\beta}}{d s_{y}} \frac{x_{\gamma}-y_{\gamma}}{|\mathbf{x}-\mathbf{y}|^{3}}$

where $s_{x}, s_{y}$ are parametrizations and their indices $x, y$ reflect belonging to the particular line. The linking integral computes a numerical invariant widely known as the linking number. Therefore, up to the normalization factor this integral is integer-valued. The linking number shows how many times one line winds around another.
We consider a null-field solution of the Maxwell equations. As was shown in Sect. 3.3, time independent parametrization of the electric field lines is obtained by rescaling the fields as (29):

$\mathbf{e}=\mathbf{E} / \mathbf{E}^{2}$

In this parametrization a field line obeys the following equations:

$\frac{d \mathbf{x}(s, t)}{d s}=\mathbf{e}(\mathbf{x}(s, t), t)$
$\frac{d \mathbf{x}(s, t)}{d t}=\mathbf{p}(\mathbf{x}(s, t), t)$

The crucial property of this parametrization is that the flows along $s$ and $t$ commute:

$\frac{d \mathbf{e}(\mathbf{x}(s, t), t)}{d t}=\frac{d \mathbf{p}(\mathbf{x}(s, t), t)}{d s}$

We pick up two distinct limit cycles $\mathbf{x}\left(s_{x}, t\right), \mathbf{y}\left(s_{y}, t\right)$ and consider the corresponding linking integral. Taking into account the fact that the tangent vectors to the field lines are rescaled electric fields (37), we represent the linking integral in the form:

$\operatorname{Link}_{\mathbf{x}(t), \mathbf{y}(t)}=-\int d s_{x} d s_{y} \epsilon_{\alpha \beta \gamma} e_{\alpha}^{x} e_{\beta}^{y} \frac{\partial}{\partial x_{\gamma}} \frac{1}{|\mathbf{x}-\mathbf{y}|}$

where we simplify the notation $e_{\alpha}^{x}:=e_{\alpha}\left(\mathbf{x}\left(s_{x}, t\right), t\right)$ and $x_{\gamma}:=x_{\gamma}\left(s_{x}, t\right)$. The time dependence of the whole linking integral is encoded in the time dependence of the field lines. Time evolution of the field lines in turn is induced by the Poynting vector (38). We also used the fact that the ratio in the integrand (35) is a total derivative:

$\frac{x_{\gamma}-y_{\gamma}}{|\mathbf{x}-\mathbf{y}|^{3}}=-\frac{\partial}{\partial x_{\gamma}} \frac{1}{|\mathbf{x}-\mathbf{y}|}$

To show that the integral (40) is time independent, we calculate its time derivative:

$$
\begin{aligned}
& \frac{d}{d t} \operatorname{Link}_{\mathbf{x}(t), \mathbf{y}(t)}=\int d s_{x} d s_{y} \epsilon_{\alpha \beta \gamma}\left[\frac{d e_{\alpha}^{x}}{d t} e_{\beta}^{y} \frac{\partial}{\partial x_{\gamma}} \frac{1}{|\mathbf{x}-\mathbf{y}|}\right. \\
& \left.\quad+e_{\alpha}^{x} \frac{d e_{\beta}^{y}}{d t} \frac{\partial}{\partial x_{\gamma}} \frac{1}{|\mathbf{x}-\mathbf{y}|}+e_{\alpha}^{x} e_{\beta}^{y} \frac{d}{d t} \frac{\partial}{\partial x_{\gamma}} \frac{1}{|\mathbf{x}-\mathbf{y}|}\right]
\end{aligned}
$$

For the first two terms in the integrand we change the derivatives of time to the derivatives along the field line using the property (39). Integrating these terms by parts, we obtain the following expression:

$$
\begin{aligned}
\frac{d}{d t} \operatorname{Link}_{\mathbf{x}(t), \mathbf{y}(t)=} & \int d s_{x} d s_{y} \epsilon_{\alpha \beta \gamma}\left[e_{\beta}^{y}\left(p_{\lambda}^{x} e_{\alpha}^{x}-p_{\alpha}^{x} e_{\lambda}^{x}\right)\right. \\
& \left.-e_{\alpha}^{x}\left(p_{\lambda}^{y} e_{\beta}^{y}-p_{\beta}^{y} e_{\lambda}^{y}\right)\right] \frac{\partial}{\partial x_{\gamma}} \frac{\partial}{\partial x_{\lambda}} \frac{1}{|\mathbf{x}-\mathbf{y}|}
\end{aligned}
$$


Using the properties of the null-field solutions (11), we have an expression of the form:

$$
\begin{aligned}
\frac{d}{d t} \operatorname{Link}_{\mathbf{x}(t), \mathbf{y}(t)=} & \int d s_{x} d s_{y}\left[\left(e_{\alpha}^{x} B_{\alpha}^{y}+e_{\alpha}^{y} B_{\alpha}^{x}\right) \frac{\partial}{\partial x_{\lambda}} \frac{\partial}{\partial x_{\lambda}} \frac{1}{|\mathbf{x}-\mathbf{y}|}\right. \\
& \left.-\left(e_{\alpha}^{y} B_{\beta}^{x}+e_{\alpha}^{x} B_{\beta}^{y}\right) \frac{\partial}{\partial x_{\alpha}} \frac{\partial}{\partial x_{\beta}} \frac{1}{|\mathbf{x}-\mathbf{y}|}\right]
\end{aligned}
$$

In the first terms 3d Dirac delta function appears:

$$
\frac{\partial}{\partial x_{\lambda}} \frac{\partial}{\partial x_{\lambda}} \frac{1}{|\mathbf{x}-\mathbf{y}|}=-4 \pi \delta^{(3)}(\mathbf{x}-\mathbf{y})
$$

The last terms turn out to be total derivatives and finally we obtain the following:

$$
\begin{aligned}
& \frac{d}{d t} \operatorname{Link}_{\mathbf{x}(t), \mathbf{y}(t)}=-\int d s_{x} d s_{y} 4 \pi \delta^{(3)}(\mathbf{x}-\mathbf{y})\left(\left(\mathbf{e}^{x} \cdot \mathbf{B}^{y}\right)\right. \\
& \left.\quad+\left(\mathbf{e}^{y} \cdot \mathbf{B}^{x}\right)\right)-\int d s_{x} d s_{y}\left[\frac{d}{d s_{y}}\left(\mathbf{B}^{x} \cdot \nabla^{x}\right) \frac{1}{|\mathbf{x}-\mathbf{y}|}\right. \\
& \left.\quad+\frac{d}{d s_{x}}\left(\mathbf{B}^{y} \cdot \nabla^{y}\right) \frac{1}{|\mathbf{x}-\mathbf{y}|}\right]
\end{aligned}
$$

The first integral may contribute because of the presence of the delta function, but it is multiplied by $\mathbf{e}^{x} \cdot \mathbf{B}^{y}$ and $\mathbf{e}^{y} \cdot \mathbf{B}^{x}$ vanishing when $\mathbf{x}-\mathbf{y}=0$ due to the null-field condition (4), so the integrand is zero. The second integral is zero because the integrand is a sum of total derivatives and the integral is along the closed line. So we conclude that the linking integral of a pair of electric limit cycles is preserved under the time evolution:

$$
\frac{d}{d t} \operatorname{Link}_{\mathbf{x}(t), \mathbf{y}(t)}=0
$$

We note that the conservation of the linking integral is a consequence of the null-field condition and closeness of the lines. Our reasoning does not involve a direct verification that the field lines do not cross.

\section{Integrability}

It is a well known fact that the Gauss linking integral is connected to the electric/magnetic helicity [10]:

$$
\begin{aligned}
& h_{e}=\int_{\mathbb{R}^{3}} d \mathbf{x} \int_{\mathbb{R}^{3}} d \mathbf{y} \mathbf{E}(\mathbf{x}) \cdot \frac{\mathbf{E}(\mathbf{y}) \times(\mathbf{x}-\mathbf{y})}{|\mathbf{x}-\mathbf{y}|^{3}} \\
& h_{m}=\int_{\mathbb{R}^{3}} d \mathbf{x} \int_{\mathbb{R}^{3}} d \mathbf{y} \mathbf{B}(\mathbf{x}) \cdot \frac{\mathbf{B}(\mathbf{y}) \times(\mathbf{x}-\mathbf{y})}{|\mathbf{x}-\mathbf{y}|^{3}}
\end{aligned}
$$

These formulas are similar to the Gauss linking integral for two closed field lines (40). In this terms the helicity can be obtained by "summing" the linking integrals over all pairs of field lines:

$h_{e}=\sum_{i \neq j} \oint d s_{i} \oint d s_{j} \mathbf{E}\left(\mathbf{x}_{i}\right) \cdot \frac{\mathbf{E}\left(\mathbf{x}_{j}\right) \times\left(\mathbf{x}_{i}-\mathbf{x}_{j}\right)}{\left|\mathbf{x}_{i}-\mathbf{x}_{j}\right|^{3}}$

Beside the fact that the sum over field lines is not rigorously defined, one can think of the helicity as the average linking number of field lines. It turns out that these quantities are integrals of motion in the null-field solutions of Maxwell equations:

$\frac{d h_{e}}{d t}=\frac{d h_{m}}{d t}=\int_{\mathbb{R}^{3}} d \mathbf{x} \mathbf{E}(\mathbf{x}) \cdot \mathbf{B}(\mathbf{x})=0$

If we believe in conservation on knot/link invariants for closed field lines, then the invariants of knots/links should be connected with nontrivial integrals of motion. It would imply that the system of Maxwell equations coupled with null-field condition is actually integrable.

\section{Electromagnetic knots}

In this section we consider particular examples of solutions of the Maxwell equations that exhibit nontrivial behaviour of the field lines. In recent papers [5,6] a family of source-free finite action solutions of Maxwell equations was constructed. The solutions are interesting because the field lines are similar to knots and links. The electric and magnetic fields as functions of the coordinates are the rational functions and therefore the solutions are called rational electromagnetic fields. We note that the zeros of the denominator are complex, so the solutions do not have singularities.

\subsection{Ranada's Hopf solution}

Our main example is the celebrated Hopf-Ranada solution:

$\mathbf{E}+\mathrm{i} \mathbf{B}=\frac{1}{\left((t-\mathrm{i})^{2}-r^{2}\right)^{3}}\left(\begin{array}{c}(x-\mathrm{i} y)^{2}-(t-\mathrm{i}-z)^{2} \\ \mathrm{i}(x-\mathrm{i} y)^{2}+\mathrm{i}(t-\mathrm{i}-z)^{2} \\ -2(x-\mathrm{i} y)(t-\mathrm{i}-z)\end{array}\right)$

This solution is the first member of the family. Here we list the properties of the field lines that were observed on the computer simulations (see Fig. 5):

- All field lines are closed.

- Every field line is topologically unknot.

- Any two field lines are linked.

- Links are preserved over time (see Fig. 6)

The last observation was a hint to consider the Gauss linking integral and demonstrate this property analytically. To make an attempt to explain the other properties, we consider 

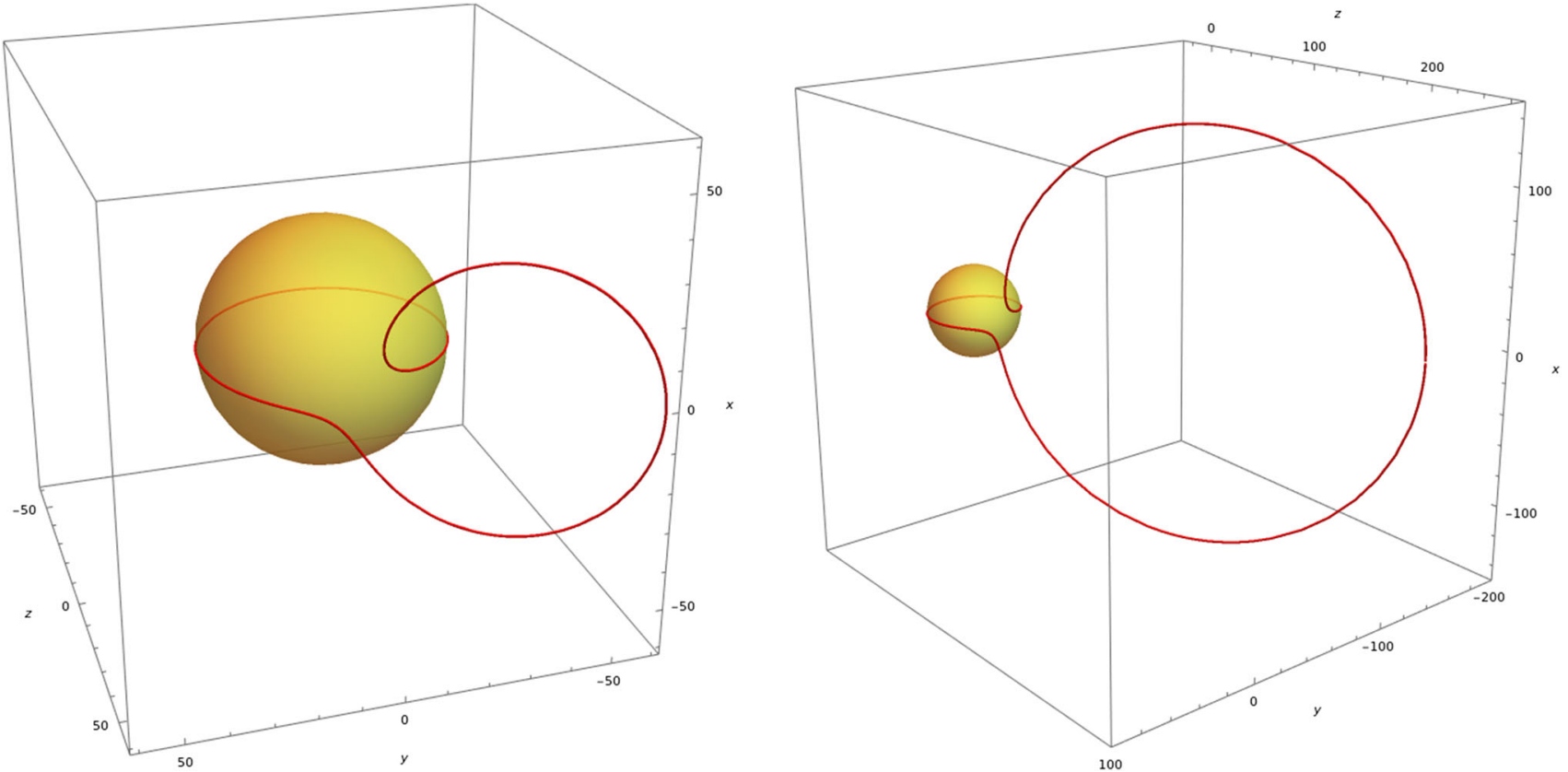

Fig. 5 The yellow sphere is the light cone $x^{2}+y^{2}+z^{2}=t^{2}$. The red lines are electric field lines at the moment $t=30$. A part of the field line lies on the equator of the sphere. The other part tends to form a circle
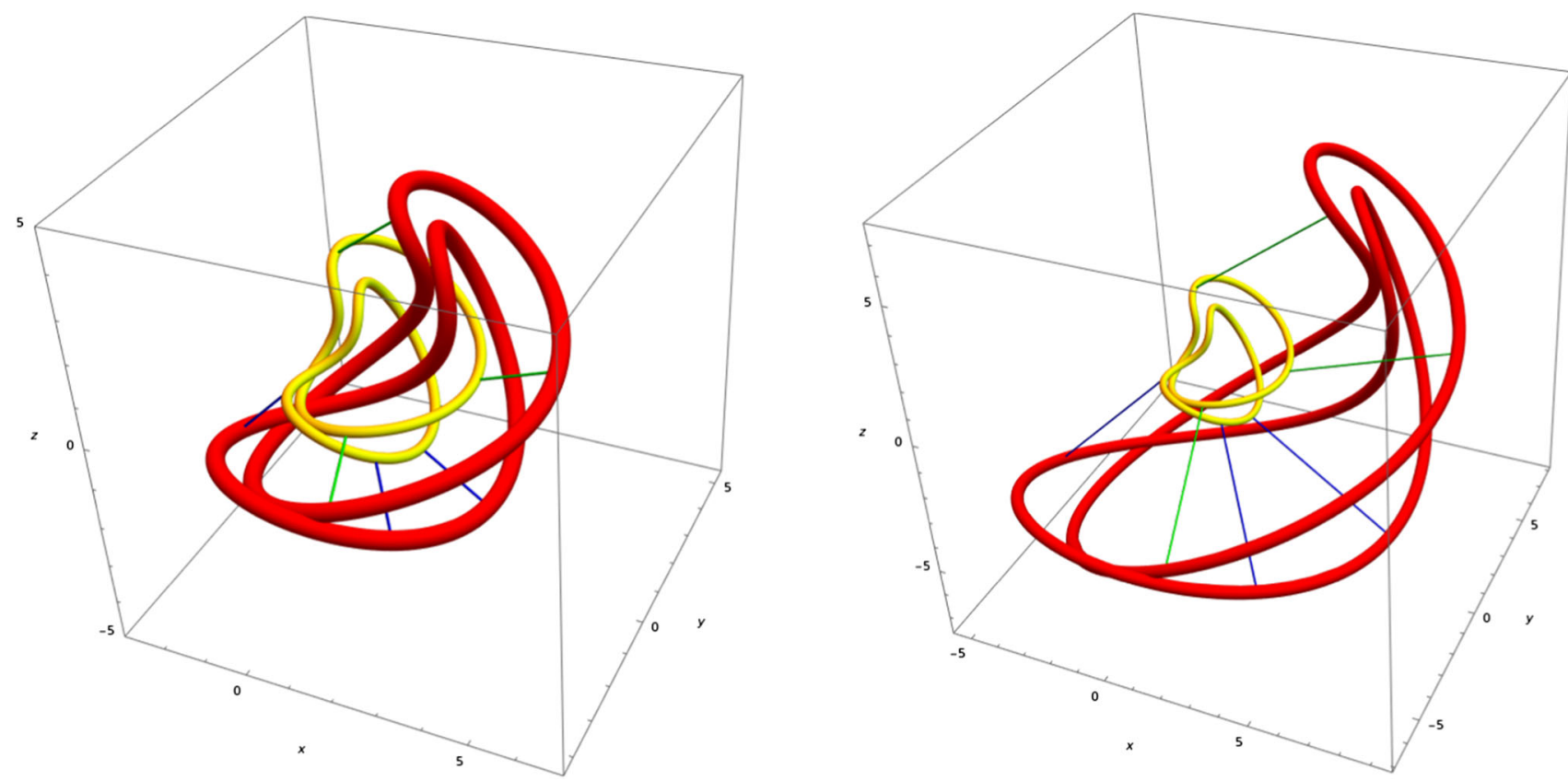

Fig. 6 The yellow lines evolve into red lines over time. There are pictures of two successive moments of time. The green and the blue curves are the trajectories. The link is preserved over time

the simplified version of the electric field, namely the electric field far from the origin. On the coordinate scales much larger that the time $t$ the electric field has the form:

$\mathbf{E}_{\infty}(\mathbf{x})=\frac{1}{r^{6}}\left(\begin{array}{c}-x^{2}+y^{2}+z^{2} \\ -2 x y \\ -2 x z\end{array}\right)$
This electric field defines an integrable system of differential equations on the field lines and the solution has the following form:

$x^{2}+(y \cos \theta+z \sin \theta-b)^{2}=b^{2}$

where all values of the radius $b$ and the angle $\theta$ are possible. The circles are normal to the plane $y z$, pass through the origin 

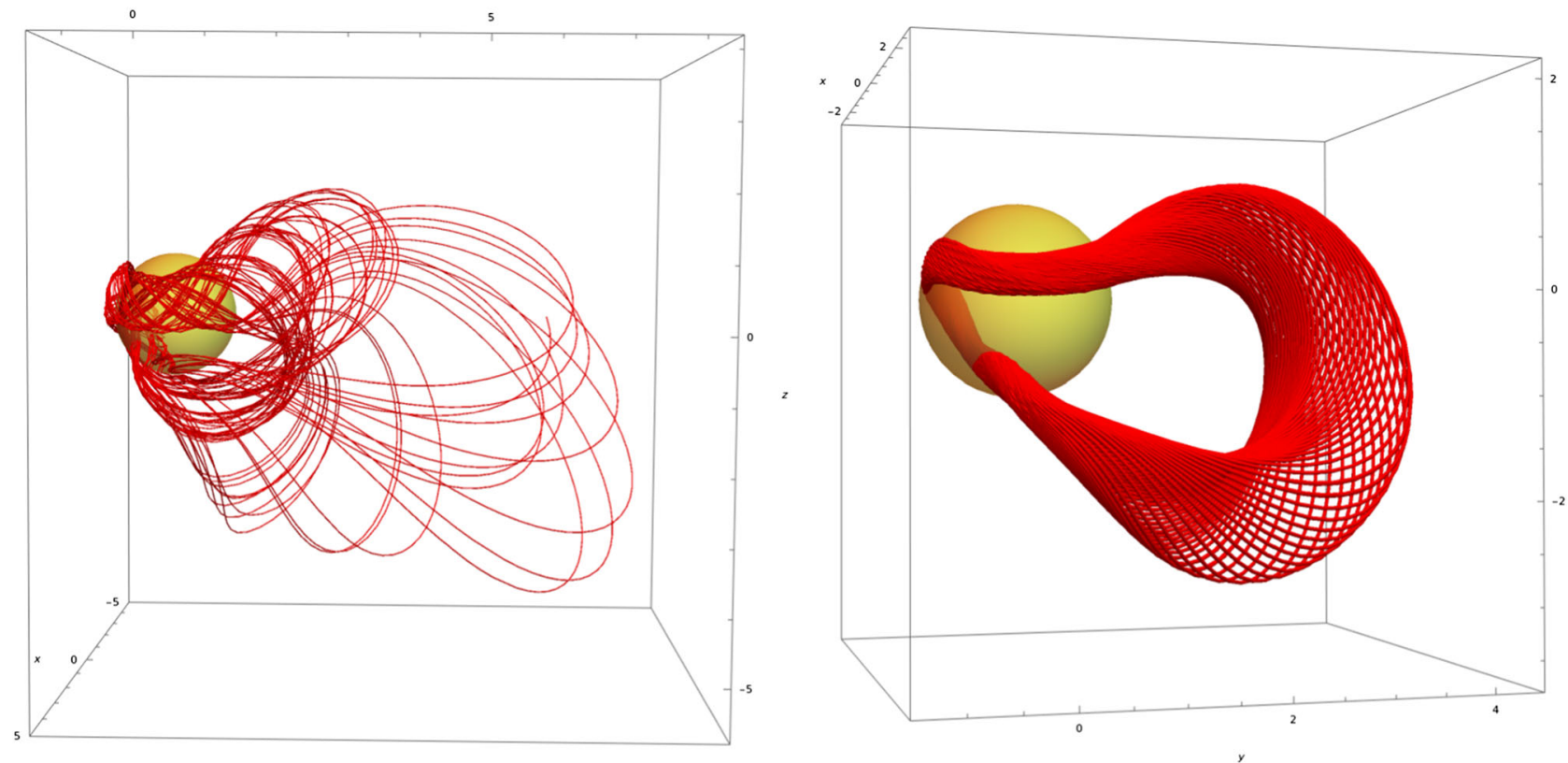

Fig. 7 The left and right pictures correspond to the same solution of the Maxwell equation. We conjecture that almost all field lines have infinite length. They can be tightly wound as in the left picture or have a form of an attractor as in the right picture

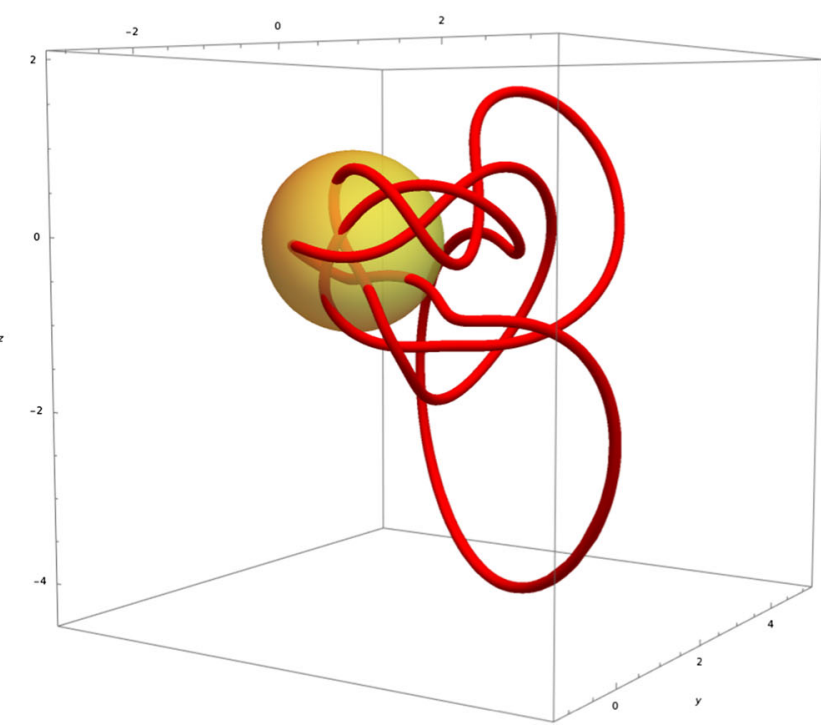

Fig. 8 These pictures correspond to the exceptional closed field lines. The left and the right field lines correspond to the same solution of the Maxwell equation. This solution is a higher member of the family. One

and their centers lie on the plane $y z$. This fact is in agreement with the Fig. 5 because one can see that a part of a field line is similar to a circle (54).

We note that the simplified version of the electric field at large scales (53) defines closed field lines (54). The fact that the simplified field at large scales defines an integrable system of differential equations and closed field lines simultaneously

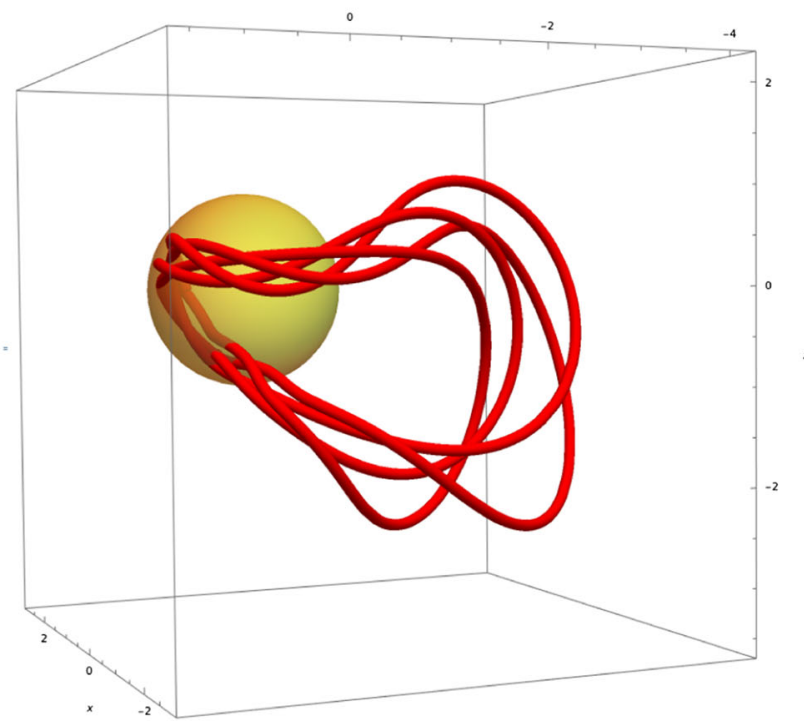

can see that the previous pictures Fig. 7 represent non-closed field lines that wind around these closed ones. The yellow sphere is the light cone $x^{2}+y^{2}+z^{2}=t^{2}$

might not be by chance. The integrability is connected with existence of the function that is constant along field lines (54):

$$
\begin{aligned}
h(\mathbf{x}) & =\frac{\sqrt{y^{2}+z^{2}}}{x^{2}+y^{2}+z^{2}} \\
\frac{d}{d s} h(\mathbf{x}(s)) & =0, \quad \text { where } \frac{d \mathbf{x}(s)}{d s}=\mathbf{E}_{\infty}(\mathbf{x})
\end{aligned}
$$


The simplified version of the field (53) is effectively two dimensional and one "integral of motion" $h(\mathbf{x})$ is sufficient to determine the field lines. In case of the full Hopf-Ranada solution two "integrals" might explain the closedness of the field lines.

\subsection{Other knots/links}

The higher members of the family of knot solutions exhibit more complicated structure of the field lines. There are rare closed limit cycles in space and they represent various knots (see Fig. 8). Almost all field lines are tightly wound around the limit cycles and we conjecture that they have infinite length (see Fig. 7).

It would be interesting to find an efficient description of this complicated structure and classify knots and links that appear in null-field solutions. For a possible approach to the problem see [6].

\section{Conclusion}

Long ago hidden integrability was predicted to be one of the governing principles for dynamics in stringy models [1114]. Since then this was proved to be the case in quite a number of examples. For a recent review of appearing of the integrable properties in various contexts see $[15,16]$. In this paper we suggest to search for integrability in generic solutions of Maxwell equations. The point is to reformulate Maxwell dynamics in terms of behavior of the field lines. Behaviour of the field lines was also discussed in [17-20]. At a given time we can define a system of non-intersecting world lines, and Maxwell dynamics convert them onto a system of world surfaces. Accordingly there are two directions $s$ and $t$ and two kinds of dynamics and, potentially, integrability for $s$ and $t$-evolutions. We provided some evidence that both kinds of integrability are really present, and $t$-integrability is related to conservation of topological invariants. It would be very interesting to develop these arguments into a full-fledged theory - most probably this will include non-Abelian considerations. One of the possible fruitful directions is establishing the connection with the rapidly developing area of knot polynomials, especially colored HOMFLY polynomials [21,22]. Secondly, at the $S U$ (2) level one can exploit peculiar properties of Lorentz and conformal groups in $4 d$ [5]. Thirdly, one can relate evolution of field lines with loop equations and integrability of eigenvalue matrix models [23-25]. We hope for a new and profound progress on these issues, which would enrich our understanding of integrability of effective theories with the help of a very concrete and down-to-earth story of Maxwell equations.
Acknowledgements We appreciate clarifying discussions with N. Kolganov, S. Mironov, V. Mishnyakov, And. Morozov, A. Popolitiov, A. Sleptsov and N. Ushakov at early stages of this project. Our work was partly supported by the grant of the Foundation for the Advancement of Theoretical Physics "BASIS" 20-1-2-36-2 (N.T.), by RFBR grants 19-02-00815 (A.M.), 20-01-00644 (N.T.), by joint RFBR grants 1951-53014-GFEN (A.M.), 19-51-18006-Bolg (A.M.), 21-51-46010-CT (A.M, N.T.).

Data Availability Statement This manuscript has no associated data or the data will not be deposited. [Authors' comment: All the existing evidence and arguments are presented in the body of the paper, thus no supplements are needed.]

Open Access This article is licensed under a Creative Commons Attribution 4.0 International License, which permits use, sharing, adaptation, distribution and reproduction in any medium or format, as long as you give appropriate credit to the original author(s) and the source, provide a link to the Creative Commons licence, and indicate if changes were made. The images or other third party material in this article are included in the article's Creative Commons licence, unless indicated otherwise in a credit line to the material. If material is not included in the article's Creative Commons licence and your intended use is not permitted by statutory regulation or exceeds the permitted use, you will need to obtain permission directly from the copyright holder. To view a copy of this licence, visit http://creativecomm ons.org/licenses/by/4.0/.

Funded by $\mathrm{SCOAP}^{3}$.

\section{References}

1. S.W. Thomson, On vortex atoms. In: Proceedings of the Royal Society of Edinburgh VI, pp. 94-105 (1867)

2. A.F. Ranada, Knotted solutions of the Maxwell equations in vacuum. J. Phys. A Math. Gen. 23(16), L815-L820 (1990). https:// doi.org/10.1088/0305-4470/23/16/007

3. A.F. Ranada, A topological theory of the electromagnetic field. Lett. Math. Phys. 18, 97-106 (1989). https://doi.org/10.1007/ BF00401864

4. J. Niemi Antti, L. Faddeev, Stable knot-like structures in classical field theory. Nature 387, 58-61 (1997). https://doi.org/10.1038/ $387058 \mathrm{a} 0$

5. O. Lechtenfeld, G. Zhilin, A new construction of rational electromagnetic knots. Phys. Lett. A 382, 1528-1533 (2018). https://doi. org/10.1016/j.physleta.2018.04.027. arXiv:1711.11144 [hep-th]

6. K. Kumar, O. Lechtenfeld, On rational electromagnetic fields. Phys. Lett. A 384, 126445 (2020). https://doi.org/10.1016/j. physleta.2020.126445. arXiv:2002.01005 [hep-th]

7. W.A. Newcomb, Motion of magnetic lines of force. Ann. Phys. 3, 347-385 (1958). https://doi.org/10.1016/0003-4916(58)90024-1

8. W.T.M. Irvine, Linked and knotted beams of light, conservation of helicity and the flow of null electromagnetic fields. J. Phys. A Math. Theor. 43, 385203 (2010). https://doi.org/10.1088/1751-8113/43/ 38/385203. arXiv: 1110.5408

9. C.F. Gauss, Zur Mathematischen Theorie der Electrodynamische Wirkungen. In: Collected Works, Vol. 5, 2nd edn. Koniglichen Gesellschaft des Wissenschaften, Gottingen, p. 601 (1833). https:// doi.org/10.1007/978-3-642-49319-5 42

10. M. Arrayas, D. Bouwmeester, J.L. Trueba. Knots in electromagnetism. Phys. Rep. 667, 1-61 (2017). ISSN:0370-1573. https://doi. org/10.1016/j.physrep.2016.11.001

11. A.Yu. Morozov, String theory: what is it? Sov. Phys. Usp. 35, 671-714 (1992). https://doi.org/10.1070/ PU1992v035n08ABEH002255 
12. A. Morozov, Matrix models as integrable systems. In: $C R M-C A P$ Summer School on Particles and Fields '94, pp. 127-210 (1995). arXiv:hep-th/9502091

13. A. Mironov. 2-d gravity and matrix models. 1. 2-d gravity. Int. J. Mod. Phys. A 9, 4355-4406 (1994). https://doi.org/10.1142/ S0217751X94001746. arXiv:hep-th/9312212

14. A. Mironov, Quantum deformations of t-functions, bilinear identities and representation theory. Electron. Res. Announc. AMS 9, 219-238 (1996). arXiv:hep-th/9409190

15. A. Andreev et al., Genus expansion of matrix models and $h$ expansion of KP hierarchy. JHEP (2020). https://doi.org/10.1007/ JHEP12(2020)038. arXiv:2008.06416 [hep-th]

16. P. Dunin-Barkowski et al., Topological recursion for the extended Ooguri-Vafa partition function of colored HOMFLY-PT polynomials of torus knots (2020). arXiv:2010.11021 [math-ph]

17. H. Jehle, Relationship of flux quantization to charge quantization and the electromagnetic coupling constant. Phys. Rev. D 3, 306345 (1971). https://doi.org/10.1103/PhysRevD.3.306

18. H. Jehle, Flux quantization and particle physics. Phys. Rev. D 6, 441-457 (1972). https://doi.org/10.1103/PhysRevD.6.441

19. H. Jehle, Flux quantization and fractional charges of quarks. Phys. Rev. D 11, 2147 (1975). https://doi.org/10.1103/PhysRevD.11. 2147
20. H. Jehle, The electron-muon puzzle and the electromagnetic coupling constant. Phys. Rev. D 15, 3727 (1977). https://doi.org/10. 1103/PhysRevD.15.3727

21. L. Bishler et al., Difference of mutant knot invariants and their differential expansion. Zh. Eksp. Teor. Fiz. 111, N9 (2020). https://doi. org/10.1134/S0021364020090015. arXiv:2004.06598 [hep-th]

22. L. Bishler et al., Distinguishing mutant knots. J. Geom. Phys. 159, 103928 (2021). ISSN:0393-0440. https://doi.org/10.1016/j. geomphys.2020.103928

23. A. Morozov, Integrability and matrix models. Phys. Usp. 37, 1-55 (1994). https://doi.org/10.1070/PU1994v037n01ABEH000001. arXiv:hep-th/9303139

24. A. Morozov, Challenges of matrix models. In: NATO Advanced Study Institute and EC Summer School on String Theory: From Gauge Interactions to Cosmology, p. 129162 (2005). https://doi. org/10.1007/1-4020-3733-3_6. arXiv:hep-th/0502010

25. A. Mironov, Matrix models of two-dimensional gravity. Phys. Part. Nucl. 33, 537-582 (2002) 